\title{
Commissioning of the RF Wien filter for a first deuteron EDM measurement at COSY / Jülich
}

\author{
Alexander Nass* \\ Institut für Kernphysik, Forschungszentrum Jülich, 52425 Jülich, Germany \\ E-mail: a.nassefz-juelich.de
}

The JEDI Collaboration ${ }^{\dagger}$ aims for a measurement of the electric dipole moments (EDMs) of deuterons and protons at the COoler SYnchrotron (COSY). To make this possible, a novel high precision waveguide RF Wien filter was installed in COSY inside a so-called low- $\beta$ section, to modulate the spin precession of the stored deuterons and protons. With this device the force of the radial electric field is canceled by the corresponding vertical magnetic force. So it is possible to directly manipulate the polarization vector of the particles without introducing any beam oscillations. The RF Wien filter has been designed to operate at harmonics of the spin precession frequency ranging from 0.6 to $1.7 \mathrm{MHz}$. For systematic investigations of sources of false EDM signals, the waveguide RF Wien filter can be rotated by $90^{\circ}$ around the beam axis. The results of several weeks of commissioning experiments at COSY with the RF Wien filter, including Lorentz force measurements, driven oscillations and the resonant build-up of vertical polarization are presented. A first test measurement similar to the upcoming first deuteron EDM measurement has been carried out as well.

23rd International Spin Physics Symposium - SPIN2018 -

10-14 September, 2018

Ferrara, Italy

\footnotetext{
*for the JEDI Collaboration: http://collaborations.fz-juelich.de/ikp/jedi

${ }^{\dagger}$ Jülich Electric Dipole moment Investigations
} 


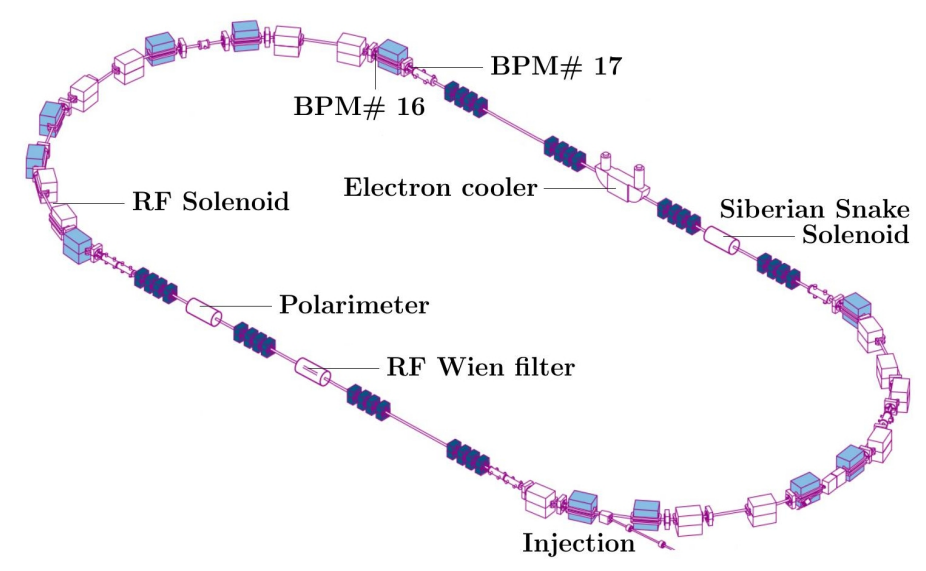

Figure 1: Sketch of COSY with the positions of the relevant elements.

\section{Introduction}

The knowledge about electric dipole moments (EDMs) of elementary particles is essential for the understanding of the formation of the universe. The question about the matter-antimatter asymmetry could be answered by the existence of an EDM outside the standard model [1] which has way too little $C P$-violation. The JEDI collaboration addresses this issue with a measurement of the EDM of Deuterons and Protons in a storage ring. For a high accuracy measurement a dedicated electrostatic storage ring is necessary [2]. On the road to that goal the collaboration uses the COoler SYnchrotron (COSY) [3] at Jülich to proof the concept, develop the technical expertise and conduct a first EDM measurement for deuterons [4].

In a future ideal storage ring for the EDM search the spin always points in the direction of the particle motion (the so-called frozen spin condition). A possible EDM would rotate the spin slowly out of the ring plane [5]. At COSY the frozen-spin condition cannot be fulfilled, therefore only a resonant build-up of the vertical polarization can lead to an EDM measurement. A novel high precision waveguide RF Wien filter $[6,7,8]$ was constructed and installed at COSY for that purpose. In the following the commissioning of that device will be described.

\section{Setup Overview}

In this experiment polarized deuterons are used in the COSY accelerator with a momentum of $970 \mathrm{MeV} / \mathrm{c}$. After beam preparation (injection, acceleration, bunching, electron cooling) the originally vertical polarization of the particles is flipped into the horizontal plane by means of an RF solenoid. The spins of the particles precess now with a frequency of $f_{\mathrm{S}}=\gamma G f_{\mathrm{rev}}=120.765 \mathrm{kHz}$, where $\gamma=1.126$ is the relativistic factor, $G=-0.143$ the deuteron's magnetic anomaly and $f_{\text {rev }}$ the revolution frequency. The polarization is measured with the former WASA detector [9], while previously the EDDA polarimeter [10] was used.

The RF Wien filter was installed in the center of one of the straight sections of the COSY accelerator (Fig. 1). Here, a so-called low- $\beta$ section is available which allows one to change the $\beta$-function of the machine and therefore the beam diameter. The driving circuit of the RF Wien 

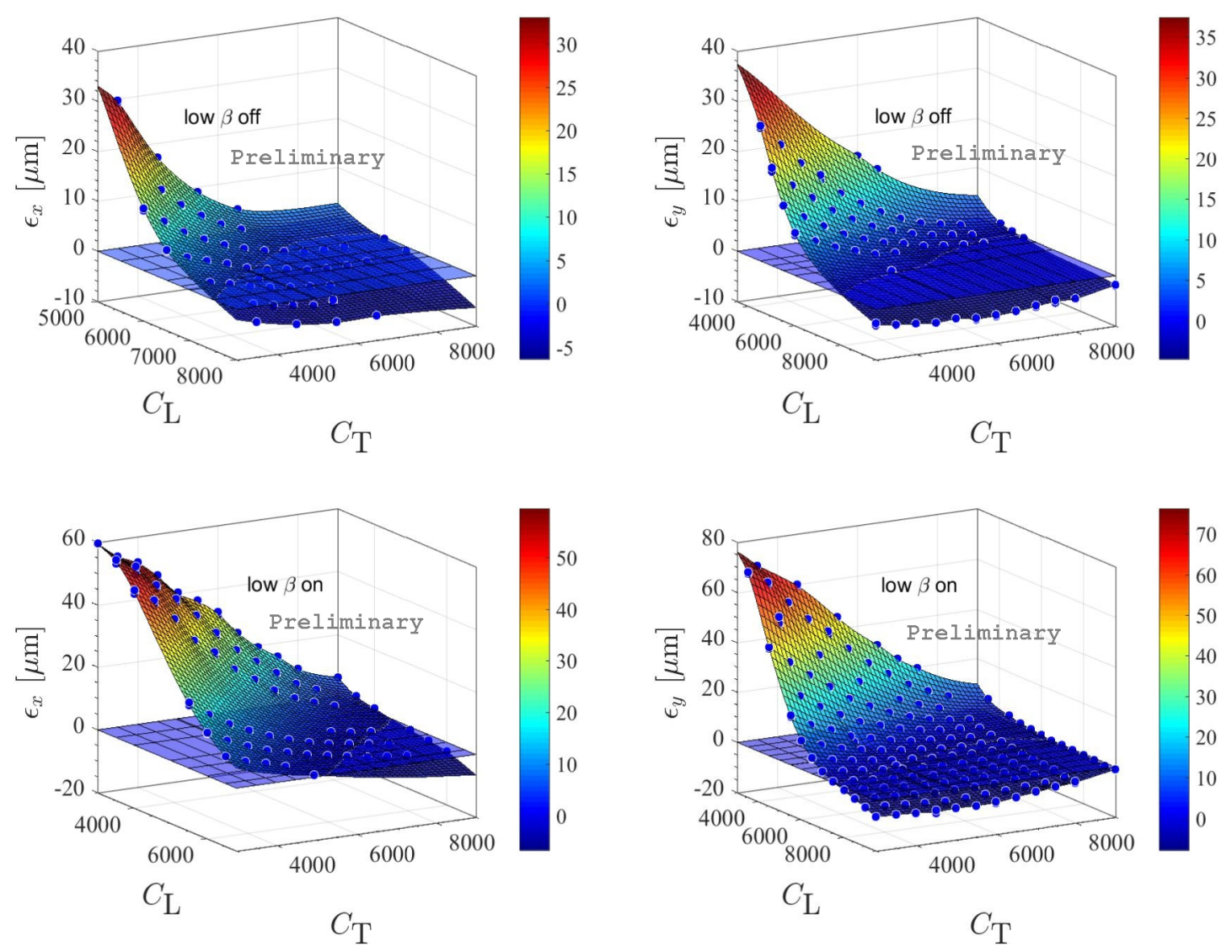

Figure 2: Horizontal (left panels) and vertical (right panels) beam oscillation amplitude $\varepsilon$ of the beam position measured at the BPM \#17 of COSY depending on the settings of the matching elements $C_{\mathrm{L}}$ and $C_{\mathrm{T}}$ at a frequency of $871 \mathrm{kHz}$ and an $R F$ power of $600 \mathrm{~W}$. The low- $\beta$ magnets were powered during the measurements shown in the lower panels. The matching point is at $C_{\mathrm{L}}=6800$ and $C_{\mathrm{T}}=4950$.

filter allows one to run at four different harmonics of the spin precession frequency ( $f_{\mathrm{WF}}=k f_{\text {rev }} \pm$ $f_{\mathrm{S}}$ with $k= \pm 1, \pm 2$ ). Adjustable capacitive elements are used to match the system in order to fulfill the Wien filter condition, i.e., induce no Lorentz force onto the circulating beam. To ensure this, the beam position is measured at the RF Wien filter frequency with a suitable beam position monitor (BPM \#17) at some other place in the accelerator. This BPM was identified using tracking simulations based on BMAD [11].

\section{Commissioning}

The measurements described in the following were performed in January / February 2018 using a beam of unpolarized deuterons with a momentum of $970 \mathrm{MeV} / \mathrm{c}$. The response of BPM $\# 17$ on the settings of the matching elements $C_{\mathrm{L}}$ and $C_{\mathrm{T}}$ at a frequency of $871 \mathrm{kHz}$ and an input power of $600 \mathrm{~W}$ (integrated electric field of $1700 \mathrm{~V}$ ) can be seen in the top two panels of Fig. 2. An additional measurement delivers the phase between the electric and magnetic field $(E / B$ phase). 

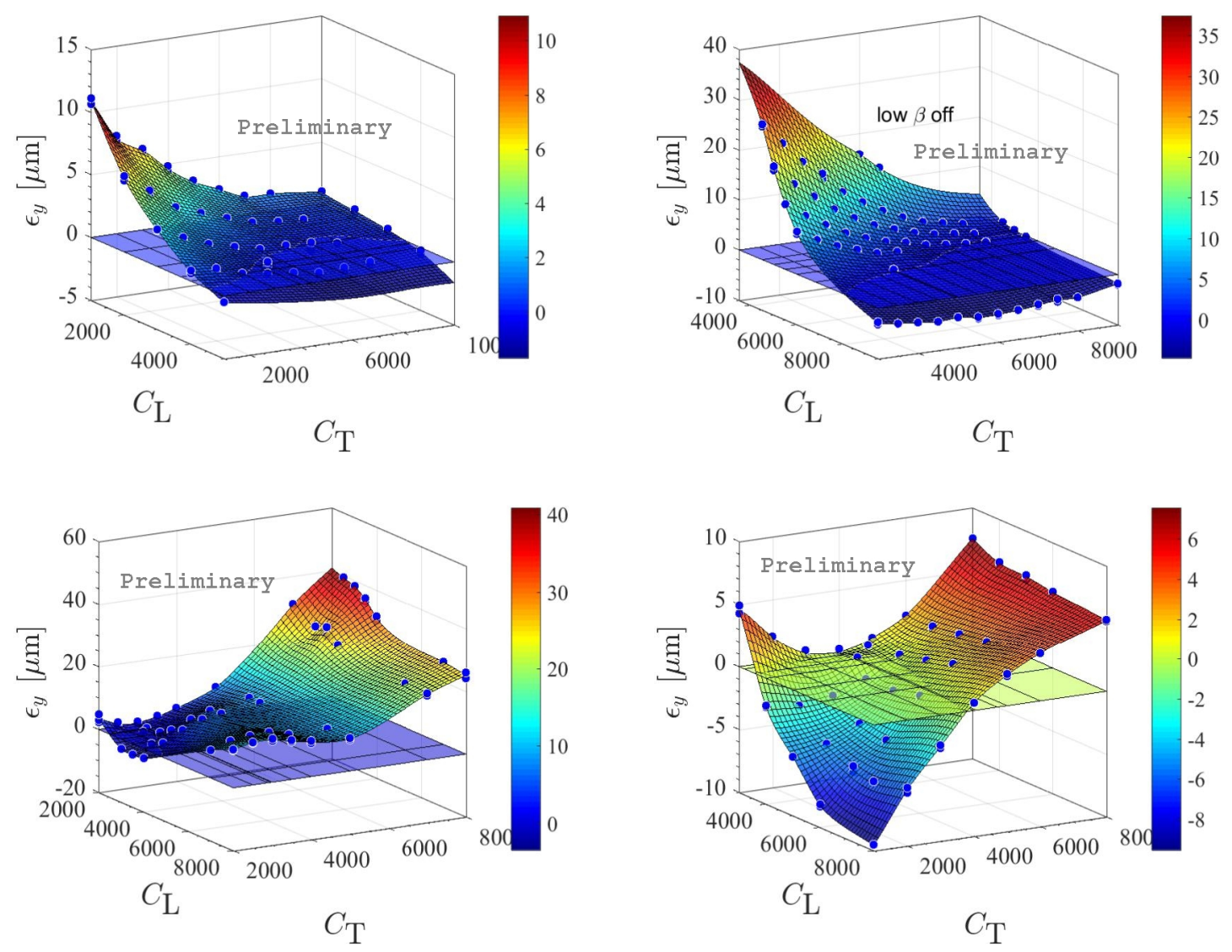

Figure 3: Vertical beam oscillation amplitude $\varepsilon$ measured at the BPM \#17 of COSY depending on the settings of the matching elements $C_{\mathrm{L}}$ and $C_{\mathrm{T}}$ with an $R F$ power of $600 \mathrm{~W}$ for four different frequencies: $629.4 \mathrm{kHz}$ (top left, matching point at $C_{\mathrm{L}}=4400$ and $C_{\mathrm{T}}=3700$ ), $871 \mathrm{kHz}$ (top right), $1379.6 \mathrm{kHz}$ (bottom left, matching point at $C_{\mathrm{L}}=2450$ and $C_{\mathrm{T}}=2980$ ) and $1621.2 \mathrm{kHz}$ (bottom right, matching point at $C_{\mathrm{L}}=$ 3200 and $\left.C_{\mathrm{T}}=4700\right)$. During these measurements the low- $\beta$ magnets were off.

This phase should also be zero to ensure a proper perfomance of the device. With this additional information the matching point can be define to be at $C_{\mathrm{L}}=6800$ and $C_{\mathrm{T}}=4950$. The lower two panels of Fig. 2 show the response of BPM \#17 with the low- $\beta$ section powered up. It can be seen that the induced beam oscillations are higher for the respective settings of $C_{\mathrm{L}}$ and $C_{\mathrm{T}}$. Powering the low- $\beta$ section on reduces the size of the deuteron beam inside the RF Wien filter at the cost of larger angles between the trajectories of the passing deuterons with respect to the axis of the RF Wien filter. This is in accordance to the calculations described in [6].

Figure 3 shows the response of the vertical beam oscillation amplitude $\varepsilon$ on the settings of the matching elements $C_{\mathrm{L}}$ and $C_{\mathrm{T}}$ at the four harmonics of the spin precession frequency which will be used in the future experiments. It can be seen that the matching points differ from each other. A feedback loop using the information of BPM $\# 17$ and the $E / B$ phase measurement will ensure that the RF Wien filter is always matched.

The next step in the commissioning was the observation of the effect of the matched RF Wien 


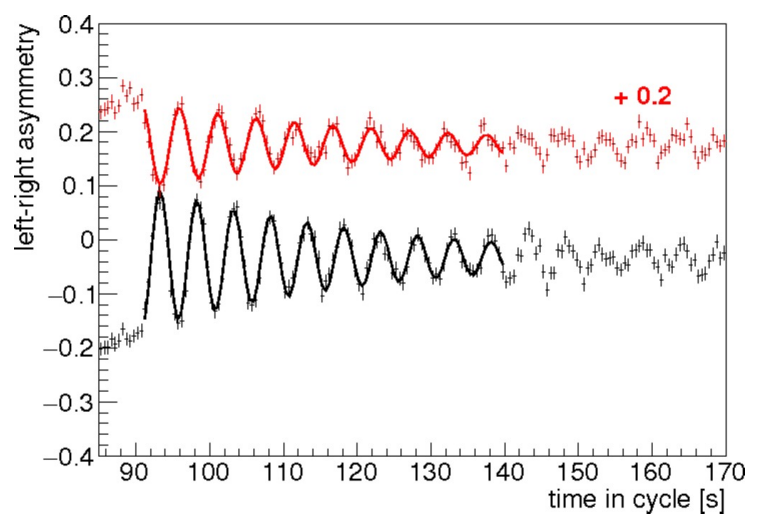

Figure 4: Oscillations of the vertical spin component of two different spin states driven by the RF Wien filter with an RF power of $15 \mathrm{~W}$ rotated into a position where the magnetic field points into the radial (horizontal) direction. The red curve is shifted for better visibility.

filter on the polarization of the deuterons. The following measurements were conducted during a measurement period in May 2018 with polarized deuterons. As described in [6] the RF Wien filter is rotatable around the COSY beam axis in order to align the magnetic field in a desired way. The device was now rotated so that the magnetic field is in the horizontal plane (radial to the circulation deuteron beam, $x$-axis). This RF field induces now small spin kicks which rotate the spin of the particles in the plane spanned by the beam direction (z-axis) and the originally vertical spin $(y$ axis). Oscillations of the vertical spin component can be observed (Fig. 4). The period length of the damped oscillations depends on the power fed into the RF Wien filter.

Rotating the RF Wien filter into a position where the magnetic field is in the vertical plane the oscillations of the spin should vanish. In an ideal ring only an oscillation (or build-up) of the vertical polarization caused by the EDM should remain. This build-up was measured depending on the relative phase between the Wien filter RF and the spin direction (Fig. 5). The left panel shows the dependence on the RF Wien filter rotation angle, where $0^{\circ}$ is the direction vertical to the COSY plane. The non-vanishing build-up at $0^{\circ}$ originates from magnetic imperfections in the COSY accelerator. These imperfections rotate the stable spin axis away from the ideal direction. To compensate for this effect in the $y z$-plane a Siberian Snake solenoid is used on the other side of the COSY accelerator. The dependence of the vertical spin build-up on the rotation angle caused by this solenoid is shown in the right panel of Fig. 5 .

To achieve a measurement of the EDM of deuterons in COSY the measured build-up rates of the vertical polarization component have to be compared with simulations of the real machine. First simulations of an ideal machine (Fig. 6) show that the EDM manifests in a minumum of the buildup rates at some rotation angle of the RF Wien filter at a solenoid strenght of zero. Simulations of the real machine including the positions of all magnets and the circulating deuteron beam together with a measurement of the dependence shown in Fig. 6 will lead to a first EDM measurement of the deuteron at COSY. 


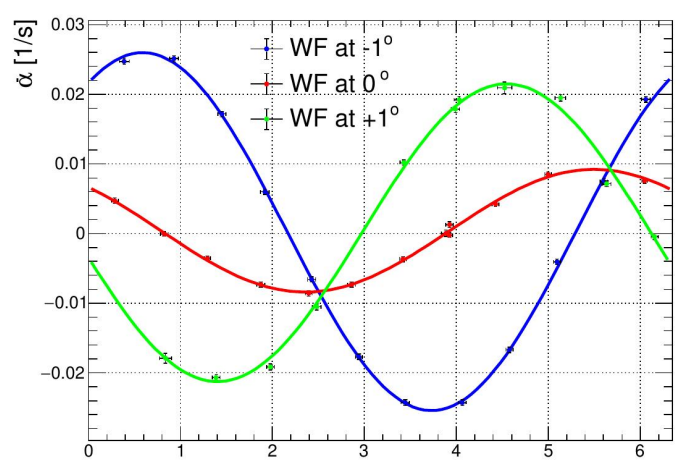

relative phase between Wien filter rf and spin direction [rad]

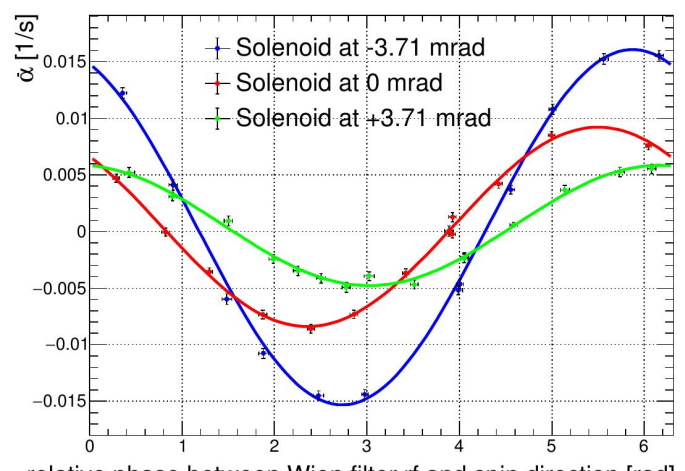

relative phase between Wien filter $\mathrm{rf}$ and spin direction [rad]

Figure 5: Build-up rates $d \alpha / d t$ of the vertical spin as a function of the relative phase between the Wien filter radio frequency and the spin direction depending on the rotation of the RF Wien filter magnetic field out of the vertical direction (left) and the rotation angle introduced by the Siberian snake solenoid (right). The RF Wien filter was powered at $1 \mathrm{~kW}$.
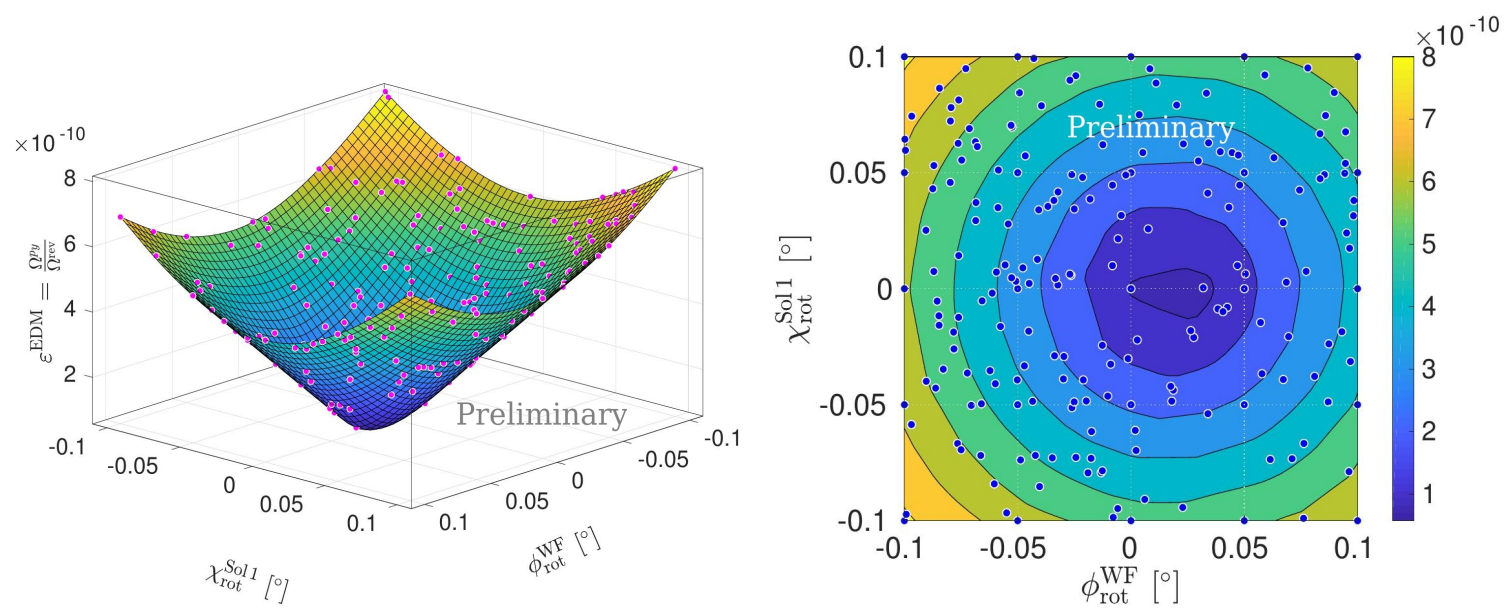

Figure 6: Resonance strength expectation $\varepsilon^{\mathrm{EDM}}=\Omega^{\mathrm{py}} / \Omega^{\mathrm{rev}}$ for an EDM of $d=10^{-18} \mathrm{e} \mathrm{cm}$, where $\Omega^{\mathrm{p}_{\mathrm{y}}}$ is the angular frequency of the vertical polarization oscillation and $\Omega^{\text {rev }}$ is the orbital angular frequency [1].

\section{Acknowledgements}

This work is supported by an ERC Advanced-Grant of the European Union (proposal number 694340).

\section{References}

[1] F. Rathmann, Proceedings of Science, talk at this conference, to be published (2019).

[2] V. Anastassopoulos, Rev. Sci. Instr., 87, 115116 (2016).

[3] R. Maier, Nucl. Instr. Meth. Phys. Res., A 390, 1 (1997).

[4] F. Rathmann, A. Saleev, and N. N. Nikolaev, J. Phys. Conf. Ser. 447, 012011 (2013). 
[5] W. M. Morse, Y. F. Orlov, and Y. K. Semertzidis, Phys. Rev. ST Accel. Beams 16, 114001 (2013).

[6] J. Slim et al, Nucl. Instr. and Meth. A828, 116 (2016).

[7] J. Slim, F. Rathmann, A. Nass, H. Soltner, R. Gebel, J. Pretz and D. Heberling, Nucl. Instr. and Meth. A859, 52 (2017).

[8] J. Slim, F. Rathmann and D. Heberling, Phys. Rev. E96, 063301 (2017).

[9] B. Hoistad and J. Ritman, arXiv:nucl-ex/0411038, (2004)

[10] D. Albers et al., Eur. Phys. J. A22, 125 (2004).

[11] D. Sagan, Nucl. Instr. and Meth. A558, 356 (2006). 